\title{
Biallelic variants in VARS in a family with two siblings with intellectual disability and microcephaly: case report and review of the literature
}

\author{
Volkan Okur, ${ }^{1,4}$ Mythily Ganapathi, ${ }^{2,4}$ Ashley Wilson, ${ }^{1}$ and Wendy K. Chung ${ }^{1,3}$ \\ ${ }^{1}$ Department of Pediatrics, Columbia University, New York, New York 10032, USA; ${ }^{2}$ Personalized Genomic \\ Medicine, Department of Pathology \& Cell Biology, Columbia University, New York, New York 10032, USA; \\ ${ }^{3}$ Department of Medicine, Columbia University, New York, New York 10032, USA
}

Abstract Two male siblings ages 15 and $10 \mathrm{yr}$ old had similar features of intellectual disability, developmental delay, severe speech impairment, microcephaly, prematurity, and transient elevation of liver enzymes in infancy. Exome sequencing revealed one novel (c.65C $>A$; p.Ala22Asp) and one ultra-rare (c.3214T>C; p.Phe1072Leu) predicted damaging missense variant in trans in the gene encoding cytoplasmic valyl-tRNA synthetase (VARS). Biallelic variants in VARS have previously been associated with a neurodevelopmental disorder characterized by microcephaly, seizures, and cortical atrophy (NDMSCA; MIM \#617802). Although our patients have no history of seizures or cortical atrophy, we suggest that the biallelic variants in VARS p.Ala22Asp and p.Phe1072Leu in this family are likely pathogenic and associated with NDMSCA, expanding the clinical phenotype of the condition.

Corresponding author: wkc15@columbia.edu

(c) 2018 Okur et al. This article is distributed under the terms of the Creative Commons Attribution-NonCommercial License, which permits reuse and redistribution, except for commercial purposes, provided that the original author and source are credited.

Ontology terms: absent speech; appendicular hypotonia; intellectual disability, moderate; microcephaly; premature birth following premature rupture of fetal membranes

Published by Cold Spring Harbor Laboratory Press

doi: 10.1101/mcs.a003301
[Supplemental material is available for this article.]

\section{CASE PRESENTATIONS}

Index case (II-1, Supplemental Fig. 1) is a 15-yr-old male with intellectual disability (ID), severe speech impairment, acquired microcephaly, and hypotonia (Table 1). The pregnancy was unremarkable except for elevated $\alpha$-fetoprotein (AFP) levels on maternal serum screening, which were later found normal in amniotic fluid analysis. He was born at $31 \mathrm{wk}$ of gestation with normal weight (1.47 kg [25\%-50\%]), length (40.6 cm [50\%]), and head circumference $(28 \mathrm{~cm}[25 \%-50 \%])$. He was admitted to the neonatal intensive care unit (NICU) because of prematurity and spent $45 \mathrm{~d}$ in the hospital with no major complications. $\mathrm{He}$ was noted to have neonatal tooth eruption. At 3 mo of age he was severely hypotonic. He had a transient mild elevation of his liver enzymes without an identifiable etiology during infancy, which resolved spontaneously. He sat without support at 4 yr of age, started crawling at $\sim 4.5 \mathrm{yr}$ of age, and now can walk with a gait trainer but usually scoots around on his bottom. He is nonverbal. He has had no regression of his milestones nor has he had problems with weight gain or short stature, but his head circumference fell $>2$ SDs below the mean at $\sim 18$ mo of age, and he remained microcephalic afterward. He has no problems with feeding and sleeping. He has repetitive movements and rocks his legs back and forth when seated and kicks his legs repeatedly when sitting on the floor. There is no history of seizures and an

\footnotetext{
${ }^{4}$ These authors contributed equally to this work.
} 
Table 1. Clinical findings of patients with biallelic variants in VARS

\begin{tabular}{|c|c|c|c|c|c|c|}
\hline \multirow[b]{2}{*}{ Patient ID } & \multicolumn{2}{|c|}{ This study } & \multicolumn{3}{|c|}{ Karaca et al. 2015} & \multirow{2}{*}{$\begin{array}{l}\text { Stephen et al. } 2018 \\
\|-2\end{array}$} \\
\hline & $\|-1$ & II-2 & BAB3643 & BAB3186 & BAB3187 & \\
\hline Gender & Male & Male & Female & Female & Female & Male \\
\hline Age & 15-yr-old & 10-yr-old & $?$ & $?$ & $?$ & 32-mo-old \\
\hline Genotype & $\begin{array}{l}\text { p.Ala22Asp } \\
\text { p.Phe1072Leu }\end{array}$ & $\begin{array}{l}\text { p.Ala22Asp } \\
\text { p.Phe1072Leu }\end{array}$ & $\begin{array}{l}\text { p.Arg1058Gln } \\
\text { p.Arg1058Gln }\end{array}$ & $\begin{array}{l}\text { p.Leu885Phe } \\
\text { p.Leu885Phe }\end{array}$ & $\begin{array}{l}\text { p.Leu885Phe } \\
\text { p.Leu885Phe }\end{array}$ & $\begin{array}{l}\text { c. } 1577-2 A>G \\
\text { p.Met1064lle }\end{array}$ \\
\hline Intellectual disability & Yes & Yes & Yes & Yes & Yes & Yes \\
\hline Speech & Nonverbal & Nonverbal & NA & NA & NA & Nonverbal \\
\hline Microcephaly (congenital vs. acquired) & Yes (acquired) & Yes (NA) & Yes (NA) & Yes (NA) & Yes (NA) & Yes (congenital) \\
\hline Seizures & No & No & Yes & Yes & Yes & Yes \\
\hline Hypotonia & Yes & Yes & NA & NA & NA & Yes \\
\hline Cortical atrophy & No & NA & Yes & Yes & Yes & Yes \\
\hline \multicolumn{7}{|l|}{ Other } \\
\hline Prematurity & Yes & Yes & NA & NA & NA & No \\
\hline Elevated liver enzymes & Yes & Yes & NA & NA & NA & No \\
\hline Stereotypic behavior & Yes & No & NA & NA & NA & No \\
\hline Neonatal tooth & Yes & No & NA & NA & NA & No \\
\hline Volvulus & No & Yes & NA & NA & NA & No \\
\hline Dysmorphic features & Yes & No & NA & NA & NA & No \\
\hline
\end{tabular}

NA, not available.

overnight ambulatory EEG at age 4-5 yr showed no epileptiform discharges. A brain MRI was also found normal. Previous genetic studies including karyotype, chromosomal microarray, fragile X, MECP2 sequencing, and metabolic screening (urine organic acids, very long chain fatty acids, and lysosomal storage disorders) were all normal.

Case 2 (II-2, Supplemental Fig. 1) is the 10-yr-old brother of the proband, who is similarly affected with ID, severe speech impairment, microcephaly, hypotonia, transient elevation of liver enzymes in infancy, and prematurity. He was born at 27 wk of gestation with intestinal volvulus which was repaired surgically in the first week of life. He has always been underweight, and his normalized height has just recently started to decline. He is also nonverbal and nonambulatory.

There is no history of consanguinity between parents and the remaining family history is unremarkable. The parents are both of European ethnicity.

\section{VARIANT INTERPRETATION}

Our patients are compound heterozygous for one novel and one ultra-rare missense variant, NM_006295:c.65C>A:p.Ala22Asp and NM_006295:c.3214T>C:p.Phe1072Leu, in the gene encoding cytoplasmic valyl-tRNA synthetase protein (VARS). Although the p.Ala22Asp variant is not present in any population databases, p.Phe1072Leu is observed in one NonFinnish European allele in gnomAD. Computational prediction algorithms favor pathogenicity for both variants. Variants are evaluated as of uncertain significance (VUS) according to American College of Medical Genetics and Genomics (ACMG) variant interpretation guidelines fulfilling PM3_Supporting, PP1, and PP4 criteria (Richards et al. 2015), although because of the overlapping clinical features to the previously reported cases they are likely 
Table 2. Genomic findings and variant interpretation

\begin{tabular}{lllllll}
\hline Gene & Genomic location & HGVS cDNA & HGVS protein & Zygosity & Parent of origin & Variant interpretation \\
\hline VARS & Chr 6:31762930 (hg19) & c.65C>A & p.Ala22Asp & Heterozygous & Maternal & Variant of uncertain significance \\
& Chr 6:31795153 (hg38) & & & & \\
VARS & $\begin{array}{l}\text { Chr 6:31747459 (hg19) } \\
\text { Chr 6:31779682 (hg38) }\end{array}$ & c.3214T>C & p.Phe1072Leu & Heterozygous & Paternal & Variant of uncertain significance \\
& & & & & & \\
\hline
\end{tabular}

causative of the patients' phenotypes (Table 2). No other likely pathogenic variants were identified that segregated with the phenotype in the family.

Aminoacyl-tRNA synthetases catalyze the aminoacylation of tRNA by their cognate amino acid. There are 37 aminoacyl-tRNA synthetases (18 cytoplasmic, 17 mitochondrial, 2 bifunctional) in human cells, and biallelic variants in many aminoacyl-tRNA synthetases have been reported in individuals with neurodevelopmental disorders (Meyer-Schuman and Antonellis 2017). Variants in VARS were first identified in patients with microcephaly, ID, and cortical atrophy (Karaca et al. 2015), and a recent study reported additional variants in patients with similar clinical findings associated with biallelic variants in VARS (Table 1; Stephen et al. 2018). VARS has three important conserved domains: glutathione S-transferase, carboxy-terminal domain (GST_C), tRNA synthetases class I (tRNA_synt_1), and anticodon-binding domain of tRNA (Anticodon_1). Four predicted likely pathogenic variants reported in the literature and in ClinVar are missense variants, and they all are located in either tRNA_synt_1 or Anticodon_1 domain. The only reported variant that is located outside of those domains is a canonical splice site variant (Fig. 1). The p.Phe1072Leu variant is also

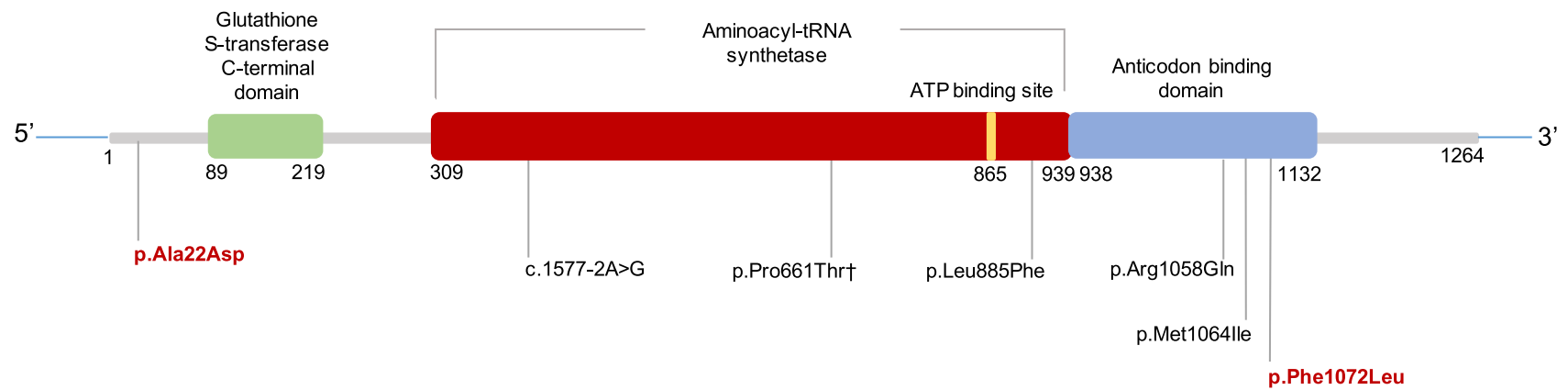

HUMAN 18 ALIAARYGEAG $28 \ldots 660$ VPLCN $665 \ldots 880$ ISLQGLHNQLL $890 \ldots 1050$ YTCLDVGLRLLSPFMPFVTEELFQRLPRRMP 1080 PANTR 18 ALIAARYGEAG $28 \ldots 660$ VPLCN $665 \ldots 880$ ISLQGLHDQLL $890 \ldots 1050$ YTCLDVGLRLLSPFMPFVTEELFQRLPRRMP 1080 MACMU 18 ALIAARYGEAG $28 \ldots 660$ VPLCN $665 \ldots 880$ VSLQGLHDQLL $890 \ldots 1050$ YTCLDVGLRLLSPFMPFVTEELFQRLPRRMP 1080 MOUSE 18 ALIAARYGEAG $28 \ldots 659$ VPLCN $664 \ldots 679$ VSLQGLYDQLL $889 \ldots 1049$ YTCLDVGLRLLSPFMPFVTEELFORLPRRTP 1079 CANLF 18 ALIAARYGEAG $28 \ldots 660$ VPLCN $665 \ldots 880$ VSLQGLHDQLL $890 \ldots 1050$ YTCLDVGLRLLSPFMPFVTEELFORLPRRTL 1080 LOXAF 18 ALIAARYGEAG $28 \ldots 658$ VPLCN $663 \ldots 878$ VSLQGLHDQLL $888 \ldots 1048$ YTCLDVGLRLLSPFMPFVTEELFQRLPRRTP 1078 RAT 18 ALIAARYGEAG $28 \ldots 660$ VPLCN $665 \ldots 880$ VSLQGLHDQLL $890 \ldots 1050$ YTCLDVGLRLLSPFMPFVTEELFQRLPRRTP 1080

Figure 1. Distribution of reported VARS variants in the literature and ClinVar over 2D representation of VARS domains given on InterPro for P26640 (SYVC_HUMAN) and the mutated residues' sequence alignment across species. Mutated residues are shown in bold black font for previously reported variants and in bold red font for variants reported in our study. HUMAN (P26640); Homo sapiens, PANTR (H2OSM5); Pan troglodytes (chimpanzee), MACMU (G7MRK4); Rhesus macaque; MOUSE (G3UY93); Mus musculus; CANLF (E2RTJ7), Canis lupus familiaris (dog); LOXAF (G5E7F5), African elephant; RAT (Q04462), Rattus norvegicus. ${ }^{\dagger}$ This variant is deposited into ClinVar but not reported in any publication. 
COLD SPRING HARBOR Molecular Case Studies
VARS variants in a neurodevelopmental disorder

located in the Anticodon_1 domain (SIFT: Damaging; PolyPhen-2: Benign; CADD v1.3: 24.8). The p.Ala22Asp variant is not located in any of the domains, but the prediction scores for this variant are supportive of pathogenicity (SIFT: Damaging; Polyphen-2: Damaging; CADD v1.3: 32). All of the previously reported variants and the variants we report are located in highly conserved residues (Fig. 1).

\section{SUMMARY}

Here we report biallelic missense variants in VARS in two similarly affected siblings with ID, severe speech impairment, acquired microcephaly, hypotonia, prematurity, and transient elevation of liver enzymes in infancy, consistent with previously reported findings in patients with biallelic VARS variants. Our patients did not have seizures or cortical atrophy, and this is different from some other reported patients in the literature, expanding the phenotypic spectrum of NDMSCA. Our patients share overlapping clinical features reported in other autosomal recessively inherited aminoacyl-tRNA synthetase disorders (i.e., IARS; MIM \#617093, LARS; MIM \#615438) including elevated liver enzymes along with microcephaly and hypotonia. Future clinical and functional studies are needed to better define the genotype-phenotype correlations and assess molecular mechanism of disease.

\section{METHODS}

Clinical exome sequencing was performed with peripheral blood DNA samples from the two affected brothers and their mother and was nondiagnostic. Raw data was requested from the diagnostic laboratory and reanalysis was performed. Variants were filtered by their population allele frequencies in Exome Aggregation Consortium (ExAC) (Lek et al. 2016), Exome Sequencing Project (ESP; http://evs.gs.washington.edu/EVS/), 1000 Genomes Samples (Auton et al. 2015), and Genome Aggregation Database (gnomAD) (Lek et al. 2016) using $1 \%$ and $3 \%$ thresholds for autosomal dominant and autosomal recessive inheritance models, respectively. Biallelic predicted pathogenic variants that fit to an autosomal recessive inheritance pattern were identified. Variants were confirmed by Sanger sequencing, and they segregated with the affected individuals in the family (Supplemental Fig. 1).

\section{ADDITIONAL INFORMATION}

\section{Data Deposition and Access}

All sequence data and interpreted variants have been deposited in ClinVar (https://www. ncbi.nlm.nih.gov/clinvar/) under accession numbers SCV000803173 (for c.65C>A) and SCV000803174 (for c.3214T>C).

\section{Ethics Statement}

Written informed consent was obtained for all individuals in this study. The study is approved by the Institutional Review Board of Columbia University under protocol \#AAAJ8651.

\section{Acknowledgments}

We thank the patients and the family for participating in this study. We thank Patricia Lanzano, Jiangyuan Hu, and Charles LeDuc for study management and Sanger sequencing confirmations. 
COLD SPRING HARBOR Molecular Case Studies
VARS variants in a neurodevelopmental disorder
Competing Interest Statement

The authors have declared no competing interest.

Received June 26, 2018; accepted in revised form July 24, 2018.

\section{Author Contributions}

V.O. and M.G. analyzed the data and drafted and critically reviewed the manuscript. A.W. provided the clinical data and critically reviewed the manuscript. W.K.C. conceived of the study, provided clinical data, analyzed the data, and drafted and critically reviewed the manuscript.

\section{Funding}

This work was supported in part by a grant from Simons Foundation and the JPB Foundation.

\section{REFERENCES}

Auton A, Brooks LD, Durbin RM, Garrison EP, Kang HM, Korbel JO, Marchini JL, McCarthy S, McVean GA Abecasis GR. 2015. A global reference for human genetic variation. Nature 526: 68-74.

Karaca E, Harel T, Pehlivan D, Jhangiani SN, Gambin T, Coban Akdemir Z, Gonzaga-Jauregui C, Erdin S, Bayram Y, Campbell IM, et al. 2015. Genes that affect brain structure and function identified by rare variant analyses of Mendelian neurologic disease. Neuron 88: 499-513.

Lek M, Karczewski KJ, Minikel EV, Samocha KE, Banks E, Fennell T, O'Donnell-Luria AH, Ware JS, Hill AJ, Cummings BB, et al. 2016. Analysis of protein-coding genetic variation in 60,706 humans. Nature 536: 285-291.

Meyer-Schuman R, Antonellis A. 2017. Emerging mechanisms of aminoacyl-tRNA synthetase mutations in recessive and dominant human disease. Hum Mol Genet 26: R114-R127.

Richards S, Aziz N, Bale S, Bick D, Das S, Gastier-Foster J, Grody WW, Hegde M, Lyon E, Spector E, et al. 2015. Standards and guidelines for the interpretation of sequence variants: a joint consensus recommendation of the American College of Medical Genetics and Genomics and the Association for Molecular Pathology. Genet Med 17: 405-424.

Stephen J, Nampoothiri S, Banerjee A, Tolman NJ, Penninger JM, Elling U, Agu CA, Burke JD, Devadathan K, Kannan R, et al. 2018. Loss of function mutations in VARS encoding cytoplasmic valyl-tRNA synthetase cause microcephaly, seizures, and progressive cerebral atrophy. Hum Genet 137: 293-303. 


\section{COLD SPRING HARBOR Molecular Case Studies}

\section{Biallelic variants in VARS in a family with two siblings with intellectual disability and microcephaly: case report and review of the literature}

Volkan Okur, Mythily Ganapathi, Ashley Wilson, et al.

Cold Spring Harb Mol Case Stud 2018, 4: a003301

Access the most recent version at doi: $10.1101 / \mathrm{mcs} . a 003301$
Supplementary http://molecularcasestudies.cshlp.org/content/suppl/2018/09/28/mcs.a003301.D Material C1
License This article is distributed under the terms of the Creative Commons Attribution-NonCommercial License, which permits reuse and redistribution, except for commercial purposes, provided that the original author and source are credited.
Email Alerting Receive free email alerts when new articles cite this article - sign up in the box at the Service top right corner of the article or click here.

